\title{
Análise da Lei de Biot-Savart em comparação com a força entre elementos de corrente de Ampère ${ }^{+*}$
}

Hugo Shigueo Tanaka dos Santos ${ }^{l}$

Mestrando no Programa de Pós-Graduação em Educação para a Ciência

e a Matemática - Universidade Estadual de Maringá

Daniel Gardelli ${ }^{2}$

Universidade Estadual de Maringá

Maringá - PR

\section{Resumo}

Atualmente, utilizamos a lei de Biot-Savart, e também a força de Grassmann, para estudar os efeitos do campo magnético e observamos que esta força aparentemente não satisfaz ao princípio da ação e reação. Em contrapartida, a força de Ampère sempre satisfaz a este princípio explicitamente e sempre na forma forte. O presente trabalho faz uma análise histórica do desenvolvimento destas duas equações de força, que foram desenvolvidas a partir de interpretações do experimento de Ørsted. Também faz uma análise comparativa entre as duas equações a fim de verificar se ambas fornecem o mesmo resultado e mostrar que a expressão de Grassmann, de fato, não satisfaz ao princípio da ação e reação. A força de Ampère, além de sempre obedecer ao princípio da ação e reação na forma forte, explica os fenômenos a partir da ação a distância, que é mais simples de ser observada e possui muitos outros resultados poderosos, não só no eletromagnetismo. Para realizar a comparação entre as duas abordagens, calculamos a força que um fio retilíneo infinito exerce em uma espira de material condutor, ambos com corrente.

\footnotetext{
+Analysis of Biot-Savart's law in comparison with Ampère's force between current elements

* Recebido: novembro de 2016.

Aceito: junho de 2017.

${ }^{1}$ E-mail: hshigueo@gmail.com, ${ }^{2}$ E-mail: dgardelli2@uem.br
} 
Palavras-chave: Força entre Elementos de Corrente de Ampère; Experimento de Ørsted; História da Ciência.

\begin{abstract}
Nowadays, we use Biot-Savart's Law and Grassmann's force to study the magnetic fields effects. We can observe that this force apparently do not always satisfy the principle of action and reaction. In contrast, Ampère's force always satisfies this principle explicitly and always along the straight line connecting the two currents elements. The present work presents a historic analysis of the development of these two forces, which have been developed based on interpretations of the Ørsted's experiment. We also compare these two forces in order to verify if both have the same result. We show that the Grassmann's expression, in fact, does not satisfy to the principle of action and reaction. Ampère's force not only follows the principle of action and reaction in the strongest way, but it also explains the phenomena based action at a distance, which is easier to be observed and has many other powerful results, not only in the electromagnetism. In order to compare these two approaches, we calculate the force that an infinite rectilinear wire exerts on a loop of conductive material, both with current.
\end{abstract}

Keywords: Ampère's Force Between Current Elements; Ørsted's Experiment; History of Science.

\title{
I. Introdução
}

Ampère desenvolveu sua expressão de força entre elementos de corrente para tentar explicar o fenômeno da deflexão de uma agulha imantada causada pela interação com um fio com corrente. Este fenômeno foi observado pela primeira vez por Hans Christian Ørsted (17771851), em 1820 (MARTINS, 1986). Além de Ampère, vários outros cientistas contemporâneos a ele tentaram explicar tal fenômeno, entre eles o próprio Ørsted, Michael Faraday, a dupla Jean-Baptiste Biot e Félix Savart e outros (GARDELLI, 2014).

A partir do seu estudo do experimento da deflexão da agulha, Biot e Savart chegaram a uma relação que é atualmente chamada de Lei de Biot-Savart. Esta lei, juntamente com a força de Grassmann, é usada para estudar os efeitos do campo magnético. Ampère explicou o mesmo fenômeno utilizando outra teoria, a saber, sua lei de força entre elementos de corrente. Mesmo com todos os elogios de Maxwell (1954b), Whittaker (1951) e outros à expressão de força entre elementos de corrente de Ampère, atualmente os livros didáticos, sejam eles de nível básico ou 
de nível superior, trazem apenas a lei desenvolvida pela dupla francesa e não a de Ampère (ASSIS, 1995).

A força entre elementos de corrente de Ampère foi considerada como "fórmula cardeal [mais importante] da eletrodinâmica" (MAXWELL, 1954, p. 175 apud ASSIS; CHAIB, 2011, p. 32) e o próprio Maxwell considerava André-Marie Ampère como o "Newton da Eletricidade". Ampère desenvolveu sua equação ao interpretar o fenômeno observado no experimento de Ørsted. Nesta interpretação, segundo Assis e Chaib (2011), ele teve uma ideia original, rica e muito frutífera que o levou a diversos experimentos inovadores e a diversos novos fenômenos que ninguém havia observado até então. Quanto ao estudo dos efeitos magnéticos, a força entre elementos de corrente de Ampère oferece resultados iguais aos da lei de Biot-Savart, porém ela é ignorada no estudo do eletromagnetismo. Quando a obra de Ampère é citada em algum livrotexto, aparece com distorções que não correspondem aos fatos históricos, em que usualmente é traçada uma linha de "evolução" da eletrodinâmica (CHAIB; ASSIS, 2007, p. 65-66).

A força de Ampère é equivalente à equação de Biot-Savart em conjunto com a força de Grassmann. Porém, a força de Grassmann satisfaz ao princípio da ação e reação somente em casos particulares (ASSIS, 1992, p. 57, 1995, p. 76). Ainda assim, a relação de Grassmann e a

de Ampère fornecem resultados idênticos. É possível, então, estudar situações específicas com essas duas concepções. Como por exemplo, analisar um caso como o cálculo da força devido a uma espira com corrente em um elemento de corrente. As duas abordagens, tanto a de Ampère quanto a de Biot e Savart, são completamente distintas e, mesmo assim, fornecem o mesmo resultado. Atualmente, usa-se a lei de Biot-Savart juntamente com a força de Grassmann para estudar casos como o que já foi citado.

Faz-se necessário considerar que Grassmann estudou filologia e teologia como curso superior e não possui educação formal em Física ou Matemática (ASSIS, 1995, p. 75) e, aparentemente, nunca realizou experimentos em Física ou sequer relacionados com eletrodinâmica, o que reforça o fato de que a força de Grassmann seja uma proposição puramente teórica de aplicar à Física o novo método de cálculo vetorial desenvolvido por ele (GRASSMANN, 1965).

Já Ampère, realizou vários experimentos e observou fenômenos que eram inéditos até então. $\mathrm{O}$ que explicita que Ampère não tentou aplicar a sua matemática aos fatos, mas, sim, explicar matematicamente o que observava.

Diferentemente do eletromagnetismo clássico, a eletrodinâmica de Ampère não utiliza nenhum tipo de campo para explicar suas interações. Todos os fenômenos explicados usualmente pela interação de matéria com campo podem ser explicados também pela ação a distância, isto é, utilizando a interação pura entre os elementos de corrente. Ou seja, na abordagem de Ampère, não se trata de algo imaterial interagindo com algo material, mas sim, da interação somente de matéria com matéria. 
O presente trabalho compara a eletrodinâmica de Ampère e o eletromagnetismo clássico. Para cumprir tal objetivo, fizemos uma pesquisa bibliográfica para mostrar as diferenças filosóficas e matemáticas das duas abordagens. Apesar da existência de referências que comparam ambas - como Assis (1992), Assis (1995), Bueno e Assis (2015) - não há muito material realizando o cálculo usando o tratamento amperiano e nem comparando este com os cálculos do eletromagnetismo clássico. A fim de realizar essa comparação e explicitar as diferenças entre os dois modos, calculamos a força exercida por um fio retilíneo infinito em uma espira, em que os dois são de material condutor e carregam correntes elétricas.

Acreditamos que este artigo pode servir de subsídio para o docente que desejar apresentar uma abordagem diferente do eletromagnetismo clássico em suas aulas. Ensinar uma maneira diferente de interpretar um determinado fenômeno - no caso deste trabalho, a interação entre eletricidade e magnetismo - é de extrema importância para auxiliar na desconstrução da imagem de uma Ciência “[...] fragmentada, criada apenas por 'mentes brilhantes', não havendo mais nada para ser inventado ou descoberto, pois se encontra pronta e acabada" (CARVALHO, 2016, p. 12). De acordo com Gardelli (2004), esta visão pode fazer com que os discentes se sintam incapazes de fazer Ciência.

É importante abordar a controvérsia entre o eletromagnetismo clássico e a eletrodinâmica de Ampère para que os alunos compreendam como funciona o processo de construção da Ciência. É esperado que eles consigam compreender que não há um único método para se fazer a Ciência e que o mesmo fenômeno pode ser interpretado de várias maneiras por diferentes pessoas. Isto é, ao apresentar a controvérsia proposta neste artigo, espera-se que isso auxilie os alunos a perceberem que a História da Ciência não é feita apenas de fatos e de conclusões tiradas destes fatos (FEYERABEND, 2011). É valido citar o prefácio de A treatise on electricity and magnetism (MAXWELL, 1954a; MAXWELL, 1954b), em que Maxwell diz que:

\begin{abstract}
De um ponto de vista filosófico, além disso, é extremamente importante que os dois métodos sejam comparados [a teoria eletrodinâmica de Ampère - e seus seguidores - que assume a ação a distância entre os corpos interagentes e o que chamamos hoje de eletromagnetismo clássico], ambos obtiveram sucesso em explicar os principais fenômenos eletromagnéticos, e ambos tentaram explicar a propagação da luz como um fenômeno eletromagnético e realmente calcularam sua velocidade, enquanto ao mesmo tempo as concepções fundamentais do que realmente ocorre, bem como a maioria das concepções secundárias das quantidades em questão, são radicalmente diferentes $^{2}$ (MAXWELL, 1954a, p. x. Tradução nossa).
\end{abstract}

\footnotetext{
2 No original, "In a philosophical point of view, moreover, it is exceedingly important that two methods should be compared, both of which have succeeded in explaining the principal electromagnetic phenomena, and both of which have attempted to explain the propagation of light as an electromagnetic phenomenon and have actually calculated its velocity, while at the same time the fundamental conceptions of what actually takes place, as well as most of the secondary conceptions of the quantities concerned, are radically different"(MAXWELL, 1954a, p. x).
} 


\section{Precedentes da observação de Ørsted}

Antes da realização e interpretações dadas ao experimento de Ørsted, não havia um consenso entre os filósofos da natureza sobre a interação entre eletricidade e magnetismo. Gardelli (2014) mostra que duas das correntes filosóficas da época interpretavam essa interação de maneira distinta: membros do Programme de Recherche Laplacian ${ }^{3}$ acreditavam que era impossível observar a interação entre fenômenos de natureza distinta; já os seguidores da Naturphilosophie acreditavam que era possível que fenômenos diferentes pudessem se relacionar, inclusive os elétricos e magnéticos.

Os integrantes do Programa de Pesquisa Laplaciano acreditavam que:

[...] os fenômenos da refração óptica, da ação capilar, da coesão dos corpos sólidos e suas propriedades cristalinas e também as reações químicas poderiam ser explicados através de uma força de atração exercida pelas moléculas constituintes da matéria (GARDELLI, 2014, p. 16).

E também tentavam explicar matematicamente todos os fenômenos físicos e matemáticos observado por filósofos experimentais.

Os integrantes do Programa de Pesquisa Laplaciano procuravam:

[...] utilizar forças intermoleculares de curto alcance agindo entre as moléculas da matéria ordinária e as moléculas dos então chamados fluidos imponderáveis, concebidos para explicar a constituição da luz, do calor, da eletricidade e do magnetismo. Eles acreditavam que as moléculas constituintes de cada fluido repeliam-se mutuamente, mas que em todos os casos, eram atraídas pela matéria ponderável. Aqueles que adotavam a teoria dos dois fluidos para explicar os fenômenos elétricos ou magnéticos, faziam a hipótese adicional de que deveria haver uma força de atração entre as moléculas dos fluidos elétricos vítreo e resinoso, assim como uma força de atração entre as moléculas dos fluidos magnéticos austral e boreal. No entanto, para os membros desta sociedade, não poderia haver qualquer interação entre dois fluidos imponderáveis de naturezas diferentes (GARDELLI, 2014, p. 19).

Já os integrantes da Naturphilosophie acreditavam que na natureza as forças fundamentais estariam sempre em conflito, o que daria origem a todos os fenômenos observados. Essa corrente filosófica teve como um de seus pioneiros Immanuel Kant (1724-1804), que questionou os fundamentos da física newtoniana. Ele se preocupava em mostrar que não é possível alcançar as "coisas como realmente são, ou as "coisas-em-si'” (GARDELLI, 2014, p. 21). No final do século XVIII, Kant havia sugerido a ideia de que a força deve se manifestar na matéria basicamente de duas formas: como força de repulsão, o que explicaria a impenetrabilidade dos

\footnotetext{
3 Programa de Pesquisa Laplaciano.
} 
corpos, assim como sua própria existência, ao impedir sua aniquilação; e como força de atração, o que definiria os limites de um corpo.

Em seu trabalho de 1799, intitulado Fundamentals of the metaphysics of nature ${ }^{4}$, Ørsted mostra que é adepto das ideias de Kant na sua introdução sobre as forças básicas da natureza (atração e repulsão) como condições necessárias para a existência da matéria de tamanho finito:

\begin{abstract}
A força expansiva evita que a força atrativa reduza a extensão da matéria a zero, e a força atrativa evita que a força expansiva dê à matéria uma extensão infinitamente grande. Elas trabalham em oposição uma à outra e produzem movimentos em direções opostas de modo que uma pode ser considerada como negativa enquanto a outra pode ser considerada como positiva (ØRSTED apud MARTINS, 2007, p. 33).
\end{abstract}

A Naturphilosophie influenciou profundamente o trabalho de muitos cientistas do século XIX, entre eles Ørsted, que estudou a interação entre efeitos elétricos e magnéticos.

\title{
III. Realização do experimento de Ørsted
}

Hans Christian Ørsted partiu do pressuposto de que na natureza haveria um eterno conflito de forças e que quando a tensão de uma manifestação de forças fosse muito intensa, ela provocaria o aparecimento de outro fenômeno. Por exemplo, caso a eletricidade fosse muito intensa, ela poderia irradiar calor e luminosidade. Ørsted supôs que o conflito elétrico seria o responsável por irradiar para todos os lados calor, luminosidade e também o magnetismo, quando se transmite uma grande quantidade de eletricidade (GARDELLI, 2014).

Ao contrário de muitos livros didáticos (CHAIB; ASSIS, 2007) que mostram Ørsted como alguém que descobriu acidentalmente a interação entre a eletricidade e a agulha de uma bússola, ele já havia previsto, em 1812, a existência do fenômeno eletromagnético, mas acreditava que, para que fosse possível observá-lo, era preciso confinar o conflito elétrico dentro de fios muito finos. Quando o conflito se tornasse muito intenso, não permaneceria mais dentro do fio e se espalharia por todo o espaço ao redor.

Em 1820, Ørsted observou o efeito que um fio com corrente causava na agulha de uma bússola. Ele aproximou um fio com corrente de uma agulha magnetizada e observou que a agulha, que estava alinhada com o polo norte terrestre, mudava de direção em sentido a oeste conforme se aproximava o fio por cima do plano da agulha. Também observou que, caso o fio fosse aproximado por baixo do plano da agulha, esta seria defletida para a direção oposta, isto é, para leste.

O experimento foi descrito pelo próprio Ørsted da seguinte forma:

Os terminais opostos do aparelho galvânico são unidos por um fio metálico, que, por concisão, chamaremos de condutor de conexão ou fio de conexão. Atribuiremos o nome de conflito elétrico ao efeito que se manifesta nesse condutor e no espaço que

\footnotetext{
${ }^{4}$ Fundamentos da metafísica da natureza.
} 
o cerca. A parte retilínea desse fio é colocada em posição horizontal, suspensa acima da agulha magnética, e paralela a ela. Se for necessário, o fio de conexão pode ser dobrado para que uma parte dele assuma a posição correta necessária à experiência. Nessa situação, a agulha magnética será movida, e a sua extremidade que está sob a parte do fio de conexão mais próxima ao terminal negativo do aparelho galvânico será desviada para oeste.

Se a distância entre o fio de conexão e a agulha magnética não exceder 3/4 de polegada, o desvio da agulha fará um ângulo de cerca de $45^{\circ}$. Se a distância variar, o ângulo diminuirá à medida que a distância cresça. Além disso, o desvio depende da eficácia do aparelho.

[...] Se o fio de conexão é colocado em um plano horizontal sob a agulha magnética, todos os efeitos são como no plano acima, mas em direção inversa. Pois o polo da agulha magnética sob o qual está a parte do fio de conexão que está próximo ao terminal negativo do aparelho galvânico desvia-se para leste (ØRSTED, 1986, p. 116-120).

O experimento pode ser representado conforme a Fig. 1. Em (a) e (b), a agulha está alinhada com o meridiano magnético, sendo que não há corrente no fio; em (c), o polo norte da agulha está defletido para a esquerda.

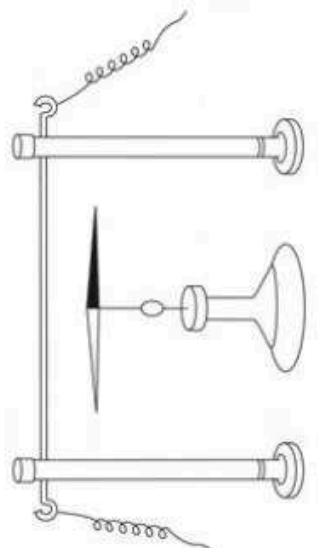

(a)

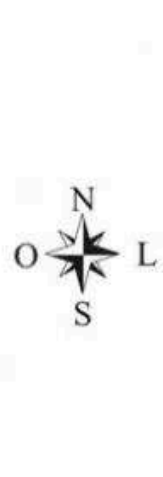

(b)

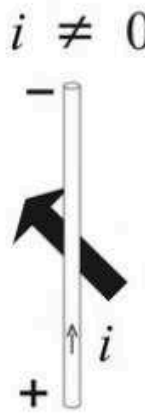

(c)

Fig. 1 -Representação do Experimento de Ørsted. Fonte: CHAIB, 2009, p. 25.

\section{O eletromagnetismo clássico}

\section{IV.1 Desenvolvimento da Lei de Biot-Savart}

Além do próprio Ørsted e outros, os franceses Jean-Baptiste Biot (1774-1862) e Félix Savart (1791-1841) também tentaram explicar o fenômeno da deflexão da agulha magnetizada devido a um fio com corrente. A explicação que a dupla deu ao experimento foi apresentada à 
Academia de Ciências de Paris no dia 30 de outubro de 1820. No trabalho, Biot e Savart concluíram que o torque magnético exercido pelo fio sobre a agulha varia com o inverso da distância entre o fio e agulha. Tal fato foi expresso em função da força magnética que o fio exercia sobre as moléculas magnéticas da agulha. É importante ressaltar que em momento algum os dois mencionam a existência de campos magnéticos ao redor dos fios, ao contrário do que se mostra em vários livros didáticos (ASSIS; CHAIB, 2006). Ou seja, haveria uma relação puramente magnética entre o fio e os polos magnéticos da agulha imantada.

Eles tinham o intuito de determinar a intensidade e a direção da força magnética exercida por um fio condutor de corrente constante longo e retilíneo sobre um polo de uma agulha magnetizada. Após eliminar o efeito do magnetismo terrestre, era possível observar que a agulha ficava perpendicular ao fio com corrente e à linha reta que ligava o fio ao centro da agulha. Isto já havia sido mostrado à Academia de Ciências por Ampère, em setembro de 1820.

A interpretação do fenômeno nas palavras de Biot e Savart, escrita na $3^{\mathrm{a}}$ pessoa, foi:

Os Srs. Biot e Savart leram uma memória na seção de 30 de outubro de 1820 da Academia de Ciências cujo tema é a determinação, por medidas precisas, das leis físicas segundo as quais os fios metálicos colocados em contato com dois polos do aparelho voltaico agem sobre os corpos imantados. As experiências foram feitas suspendendo por fios de seda lâminas retangulares ou fios cilíndricos de aço temperado, imantadas pelo método de duplo contato[...]. Com o auxílio destes procedimentos os Srs. Biot e Savart foram conduzidos ao resultado seguinte que exprime rigorosamente a ação experimentada por uma molécula de magnetismo austral ou boreal colocada a uma distância qualquer de um fio cilíndrico muito fino e indefinido, tornado magnético, pela corrente voltaica. A força que atua sobre a molécula é perpendicular a esta linha e ao eixo do fio. Sua intensidade é inversamente proporcional a esta distância. A natureza de sua ação é a mesma que a ação de uma agulha imantada que fosse colocada sobre o contorno do fio em um sentido determinado e sempre constante em relação à direção da corrente voltaica; de tal maneira que uma molécula de magnetismo boreal e uma molécula de magnetismo austral seriam assim solicitadas em sentidos contrários, embora sempre seguindo a mesma [linha] reta determinada pela construção precedente (BIOT e SAVART, Nota sobre o Magnetismo da Pilha de Volta. Em: ASSIS; CHAIB, 2006, p. 307-308).

Atualmente, embora Biot e Savart nunca tenham assumido a existência de campos magnéticos, usa-se a lei de Biot-Savart para calcular o campo magnético de um fio. Em linguagem vetorial moderna, ela é da forma:

$$
d \vec{B}_{1}=-\frac{\mu_{0}}{4 \pi} \frac{1}{r_{12}^{2}}\left(i_{1} d \vec{l}_{1} \times \widehat{r}_{12}\right)
$$

Diz-se que esta lei representa o campo magnético gerado em um ponto do espaço por um elemento de corrente de um fio, a uma distância $r_{12}$ desse elemento de corrente; $\widehat{r}_{12}$ é um 
vetor unitário, que aponta do elemento de corrente para o ponto considerado; $\mu_{0}$ é a permeabilidade magnética do meio e $i_{1} d \vec{l}_{1}$ é um elemento de corrente do fio.

$\mathrm{O}$ conceito de elemento de corrente havia sido inventado por Ampère para representar um "comprimento infinitesimal do fio condutor de corrente elétrica orientado no sentido da corrente" (GARDELLI, 2014, p. 44).

\section{IV.2 Força de Grassmann}

Hermann Günther Grassmann (1809-1877), aparentemente nunca frequentou cursos de matemática ou de física na Universidade de Berlim. Apesar de ter voltado para a sua cidade natal depois de terminar o curso universitário, nunca chegou a lecionar em uma universidade, apenas nas escolas secundárias locais. Ao que parece, Grassmann nunca realizou experimentos de eletromagnetismo (ASSIS; CHAIB, 2011, p. 223).

Mesmo que, aparentemente, Grassmann não tenha feito experimentos em eletromagnetismo, ele "publicou um artigo intitulado 'Uma nova teoria da eletrodinâmica'. Em 1877, publicou outro artigo [...], no qual mostrou que a expressão de Clausius, de 1877, para a força entre elementos de corrente era a mesma que ele próprio havia publicado em 1845" (ASSIS; CHAIB, 2011, p. 223).

Atualmente, apenas a força de Grassmann aparece nos livros didáticos, sejam do ciclo básico ou em níveis avançados, mesmo com os elogios feitos à força de Ampère por Maxwell (1954b), Whittaker (1951) e outros. Em linguagem vetorial moderna, a força de Grassmann é dada por:

$$
d^{2} \vec{F}_{12}^{G}=i_{2} d \vec{l}_{2} \times d \vec{B}_{1}
$$

ou

$$
d^{2} \vec{F}_{21}^{G}=i_{1} d \vec{l}_{1} \times d \vec{B}_{2}
$$

sendo $d \vec{B}_{1}$ o campo magnético gerado pelo elemento de corrente $i_{1} d \vec{l}_{1}$, dado pela lei de BiotSavart. Esta expressão foi usada pela primeira vez por Grassmann em um artigo de 1845 (ASSIS, 1995, p. 75). Usando uma propriedade do triplo produto vetorial, é possível ver que a força de Grassmann só satisfaz ao princípio da ação e reação em casos muito particulares. Uma demonstração mais detalhada pode ser encontrada em Assis (1995) e Santos (2016). Neste sentido, há casos em que a força de Grassmann, quando se utilizam elementos de corrente, não satisfaz à terceira lei de Newton. Entretanto, Griffiths (2010, p. 242-243) mostra que, quando se assume que o campo magnético possui momento, a força de Grassmann volta a obedecer ao princípio da ação e reação, isto é, ao se fazer uma hipótese $a d$ hoc, a força de Grassmann volta a satisfazer à terceira lei de Newton. 


\section{A eletrodinâmica de Ampère}

André-Marie Ampère (1775-1836) assistiu às demonstrações do experimento de Ørsted feitas por Arago em 4 de setembro de 1820 e, a partir daí, começou a trabalhar intensamente no assunto. Para explicar o fenômeno do conflito elétrico, supôs que todos os fenômenos magnéticos já conhecidos até então poderiam ser explicados por meio da interação entre elementos de corrente. Com isso, ele assumiu que a Terra e os ímãs naturais também possuíam elementos infinitesimais de corrente. A partir de suas hipóteses, Ampère chegou a prever - e observar - vários fenômenos que ninguém havia observado até então, como, por exemplo, a interação entre dois fios com corrente (ASSIS; CHAIB, 2011).

Partindo do princípio de que coisas de mesma natureza podem interagir entre si, não seria absurdo imaginar a existência de correntes elétricas microscópicas dentro da agulha da bússola e que também fosse possível a interação entre fios com corrente (GARDELLI, 2014).

Fortemente influenciado pelas ideias de Laplace, Ampère chegou, em 1822, a uma expressão matemática que descreve a força entre dois elementos de corrente. A expressão também conseguiu explicar todas as suas observações experimentais. Em linguagem vetorial moderna e no Sistema Internacional de Unidades, pode-se representar a força entre elementos de corrente em Ampère como foi escrita por André K. T. Assis (1995):

$$
d^{2} \vec{F}_{12}^{A}=\frac{\mu_{0}}{2 \pi} \frac{i_{1} i_{2}}{r_{12}^{2}}\left[\left(d \vec{l}_{1} \cdot d \vec{l}_{2}\right)-\frac{3}{2}\left(d \vec{l}_{1} \cdot \widehat{r}_{12}\right)\left(d \vec{l}_{2} \cdot \widehat{r}_{12}\right)\right] \widehat{r}_{12}
$$

na qual $\mu_{0}$ é a permeabilidade magnética do meio.

Sabendo que o produto interno entre dois vetores é comutativo, vemos que a expressão de Ampère obedece ao princípio da ação e reação. Isto é detalhado em Assis (1995), Assis e Chaib (2011) e Santos (2016).

\section{Ampère versus Biot-Savart}

Tanto a abordagem de Biot-Savart e Grassmann quanto a abordagem de Ampère fornecem os mesmos resultados finais. Mesmo com todas as diferenças de origem e filosóficas, elas são equivalentes. Segundo Bueno e Assis (2015), a razão disso é "uma diferencial exata que, quando integrada no circuito fechado [...], resulta um valor nulo" (BUENO; ASSIS, 2015, p. 99).

Para mostrar que as duas equações levam ao mesmo resultado, calcularemos a força exercida por um fio retilíneo infinito em uma espira, ambos de material condutor e com correntes elétricas. Faremos também o cálculo da força que a espira faz no fio retilíneo, a fim de verificar se tanto a força de Ampère quanto a força de Grassmann são compatíveis com o princípio da ação e reação. 
Um caso parecido pode ser encontrado no exemplo 5.6 do Capítulo 5 da $3^{\text {a }}$ edição do livro Eletrodinâmica ${ }^{5}$, de David J. Griffiths (2010, p. 152-153), que calcula o campo magnético devido a um circuito circular usando a lei de Biot-Savart. Como a concepção amperiana não admite a existência de campos magnéticos e trata da interação pura entre elementos de corrente, calcularemos a interação da espira - ou circuito circular - com um fio retilíneo infinito, conforme a Fig. 2:

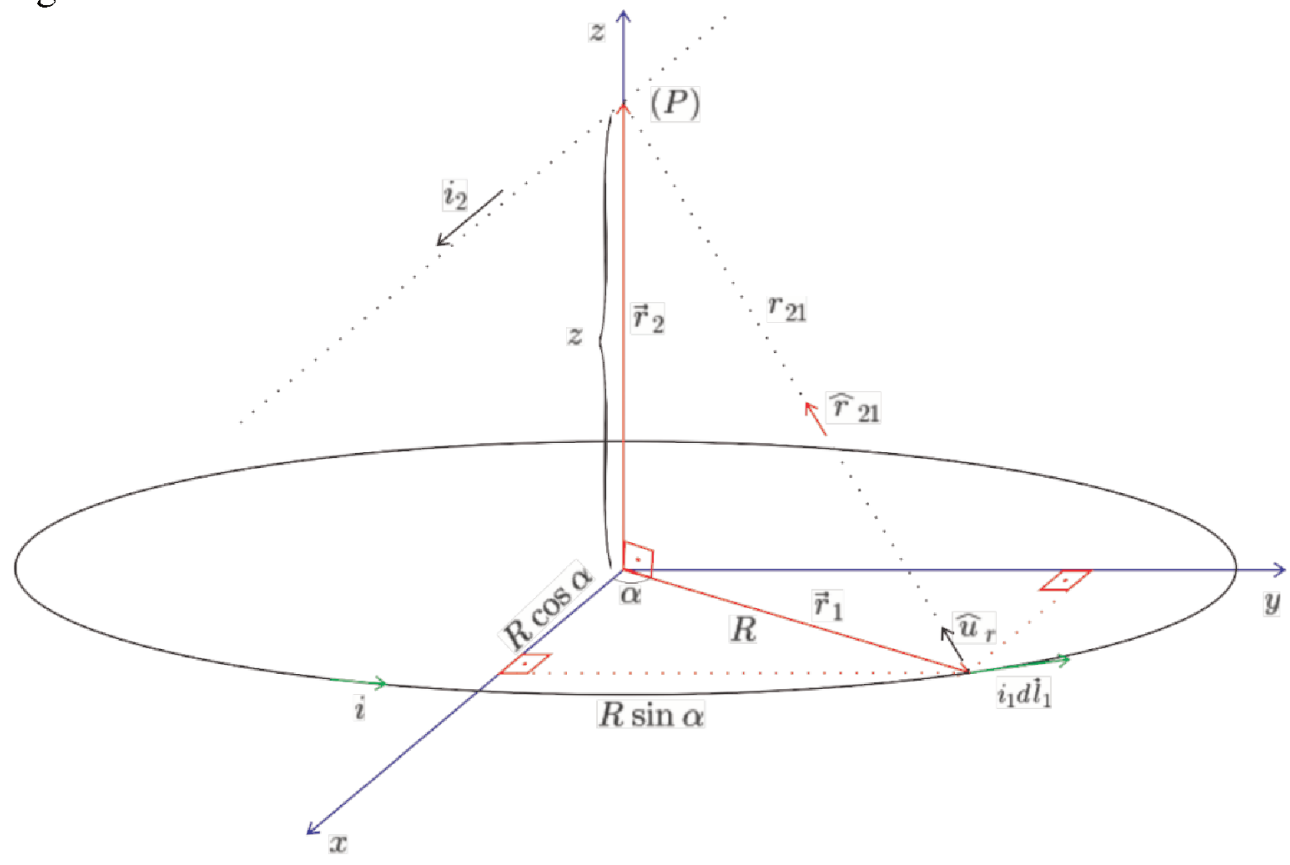

Fig. 2 - Espira com corrente interagindo com um elemento de corrente.

Na figura, cada elemento é:

$i_{1}:$ a corrente que passa pela espira;

$i_{1} d \vec{l}_{1}:$ um elemento de corrente orientado na espira;

$R$ : o raio da espira;

$\vec{r}_{1}$ : o vetor que vai da origem até o elemento de corrente $i_{1} d \vec{l}_{1}$;

$R \cos \alpha$ : a projeção do vetor $\vec{r}_{1}$ no eixo coordenado x;

$R \sin \alpha$ : a projeção do vetor $\vec{r}_{1}$ no eixo coordenado y;

$i_{2}$ : a corrente que passa a uma altura $\mathrm{z}$ do centro da espira orientada ao longo do eixo coordenado $\mathrm{x}$;

$i_{2} d \vec{l}_{2}:$ um elemento de corrente cujo centro está no ponto $(\mathrm{P})$ a uma altura $\mathrm{z}$ do centro da espira e que está orientado na direção paralela ao eixo coordenado $\mathrm{x}$

$\vec{r}_{2}$ : o vetor que vai da origem até o elemento de corrente que está em um ponto $(\mathrm{P}) ;$

5 É interessante mencionar que, apesar de o livro ter o nome Eletrodinâmica, o tratamento dado na obra não é eletrodinâmico. Como Assis e Chaib (2011) chamam atenção, este termo é usado apenas para a interação entre condutores com corrente, o que não é mostrado na obra. 
$\widehat{r}_{21}$ : o vetor unitário que vai do elemento de corrente $i_{1} d \vec{l}_{1}$ até o outro elemento de corrente no ponto $(\mathrm{P})$

$r_{21}$ : a distância entre o elemento de corrente $i_{1} d \vec{l}_{1}$ e o elemento de corrente que está a uma altura $\mathrm{z}$ do centro da espira.

\section{VI.1 Usando a Lei de Biot-Savart e a Força de Grassmann}

Sabendo que $\vec{r}_{21}=-R \cos (\alpha) \hat{\imath}-R \sin (\alpha) \hat{j}+z \hat{k}$ e substituindo este valor na lei de BiotSavart a fim de encontrar o campo magnético devido a um elemento de corrente, após resolver o produto vetorial e organizar a equação, ficamos com:

$$
d \vec{B}_{1}=\frac{\mu_{0}}{4 \pi} \frac{i_{1} R(z \cos \alpha d \alpha \hat{i}+z \sin \alpha d \alpha \hat{j}+R d \alpha \hat{k})}{\left(R^{2}+z^{2}\right)^{3 / 2}}
$$

Para calcular a força de interação entre a espira e um elemento de corrente $i_{2} d \vec{l}_{2}$ cujo centro encontra-se no ponto $(\mathrm{P})$ selecionado anteriormente, vamos usar a força de Grassmann. Neste caso, o elemento de corrente $i_{2} d \vec{l}_{2}$ está orientado na direção paralela ao eixo x. Assim, após substituir o valor infinitesimal do campo magnético na expressão de Grassmann, resolver o produto vetorial e organizar a equação, ficamos com:

$$
d^{2} \vec{F}_{12}^{G}=\frac{\mu_{0}}{4 \pi} \frac{i_{1} i_{2} R}{\left(R^{2}+z^{2}\right)^{3 / 2}}[\hat{j}(-R d \alpha)+\hat{k}(z \sin \alpha d \alpha)] d l_{2}
$$

Após integrar, aplicar os limites de integração e organizar a equação, temos que a força da espira por unidade de comprimento do fio retilíneo é dada por:

$$
\frac{d \vec{F}_{12}^{G}}{d l_{2}}=-\frac{\mu_{0}}{2} \frac{i_{1} i_{2} R^{2}}{\left(R^{2}+z^{2}\right)^{3 / 2}} \hat{j}
$$

A fim de verificar se a força de Grassmann obedece ao princípio da ação e reação, vamos usar a Equação (3). Caso a suposição seja verdadeira, devemos ter que $d^{2} \vec{F}_{12}^{G}=-d^{2} \vec{F}_{21}^{G}$.

De modo análogo ao anterior, vamos calcular, com a lei de Biot-Savart, o campo magnético de um elemento de corrente do fio retilíneo, que agora é da forma:

$$
d \vec{B}_{2}=\frac{\mu_{0}}{4 \pi} \frac{i_{2}}{\left(R^{2}+z^{2}\right)^{3 / 2}}\left(z d l_{2} \hat{j}+R \sin \alpha d l_{2} \hat{k}\right)
$$

Substituindo a Equação (8) na expressão de Grassmann e realizando as operações a fim de encontrar $d^{2} \vec{F}_{21}^{G}$, obtemos o seguinte resultado:

$$
d^{2} \vec{F}_{21}^{G}=\frac{\mu_{0}}{4 \pi} \frac{i_{1} i_{2}}{\left(R^{2}+z^{2}\right)^{3 / 2}}\left[\hat{i}\left(R^{2} \sin \alpha \cos \alpha\right)+\hat{j}\left(R^{2} \sin ^{2} \alpha\right)+\hat{k}(-R z \sin \alpha)\right] d \alpha d l_{2}
$$

Comparando as Equações (9) e (6), vemos que $d^{2} \vec{F}_{12}^{G} \neq-d^{2} \vec{F}_{21}^{G}$. Porém, ao integrar os dois lados da equação a fim de encontrar o resultado final, obtemos: 


$$
\frac{d \vec{F}_{21}^{G}}{d l_{2}}=+\frac{\mu_{0}}{2} \frac{i_{1} i_{2} R^{2}}{\left(R^{2}+z^{2}\right)^{3 / 2}} \hat{j}
$$

\section{VI.2 Usando a força entre elementos de corrente de Ampère}

Agora usaremos a expressão de Ampère para calcular a mesma interação. Após substituir os termos, resolver os produtos internos e organizar a equação, encontramos que:

$$
d^{2} \vec{F}_{12}^{A}=\frac{\mu_{0}}{2 \pi} \frac{i_{1} i_{2}}{\left(R^{2}+z^{2}\right)^{3 / 2}}\left[\hat{i}\left(R^{2} \sin \alpha \cos \alpha\right)+\hat{j}\left(R^{2} \sin ^{2} \alpha\right)+\hat{k}(-R z \sin \alpha)\right] d \alpha d l_{2}
$$

Ao integrar os dois lados da igualdade com a finalidade de se obter a força que a espira faz em um elemento de corrente do fio retilíneo, temos:

$$
\frac{d \vec{F}_{12}^{A}}{d l_{2}}=-\frac{\mu_{0}}{2} \frac{i_{1} i_{2} R^{2}}{\left(R^{2}+z^{2}\right)^{3 / 2}} \hat{j}
$$

Este resultado é igual ao fornecido pela Equação (7).

Como a expressão de Ampère depende exclusivamente de produtos internos entre vetores - que são comutativos - já é fácil perceber que $d^{2} \vec{F}_{12}^{A}=-d^{2} \vec{F}_{21}^{A}$.

\section{Considerações finais}

Partindo das duas interpretações apresentadas acima, analisamos um caso a fim de comparar a força de Ampère entre elementos de corrente com a força de Grassmann. Como vimos, a força de Ampère, de fato, sempre satisfaz ao princípio da ação e reação na forma forte, ao contrário da força de Grassmann. Porém, como mostrado por Chaib e Assis (2007), a força de Ampère não aparece em quase nenhum dos livros-texto e, quando aparece, está distorcida. O que se faz é traçar "uma linha da evolução da eletrodinâmica obedecendo à sequência Oersted, Biot-Savart, Ampère, Faraday e Maxwell. Cada um acrescentando formalmente uma determinada parcela de conhecimento para o acúmulo e desenvolvimento da ciência do eletromagnetismo" (CHAIB; ASSIS, 2007, p.66).

Além da força entre elementos de corrente de Ampère sempre obedecer à $3^{\mathrm{a}}$ lei de Newton, é interessante observar o fato de que ela usa apenas a ideia de ação a distância, evitando, desta maneira, a introdução do conceito de campo para ajudar a explicar como ocorre a interação entre dois corpos. Assis (1999) conclui que:

[...] existem muitos aspectos positivos relacionados com a ação a distância: sua simplicidade, os poderosos resultados que são obtidos com ela no eletromagnetismo e na gravitação, a implementação do princípio de Mach, o fato de que a primeira equação 
de onda descrevendo perturbações eletromagnéticas ter sido obtida a partir da ação a distância antes de Maxwell etc. ${ }^{6}$ (ASSIS, 1999, p. 55. Tradução nossa).

Além disso, Maxwell, Whittaker e outros fazem muitos elogios a Ampère, que foi considerado o "Newton da Eletricidade" por Maxwell. Ainda segundo Maxwell, a força entre elementos de corrente de Ampère é "sem dúvida, a melhor, uma vez que é a única que faz com que a força em cada um dos dois elementos [de corrente], sejam não só de mesma intensidade e sentidos opostos, mas também estejam na linha reta que os une"7 (MAXWELL, 1954, p. 174. Tradução nossa).

A ideia de Ampère é tão valiosa que, além dos elogios já citados, Wilhelm Eduard Weber (1804-1891) partiu da força de Ampère para obter sua equação de força entre cargas elétricas. A força de Weber prevê todos os fenômenos eletrostáticos e eletrodinâmicos conhecidos (ASSIS, 1992; ASSIS, 1995). Mesmo com todos esses elogios, a ideia de Ampère sofreu e sofre várias críticas. A seguir vamos discutir algumas delas.

O próprio Ørsted nunca aceitou todas as ideias de Ampère. Criticava principalmente a suposição da existência de correntes elétricas microscópicas dentro de um ímã. Além disso, Ampère acreditava que a ação entre dois condutores com corrente era direta e não rotatória como na interpretação dada por Ørsted.

Ampère era contrário às ideias de Ørsted, e também não acreditava na concepção de polos magnéticos. Por isso, a interpretação entre correntes moleculares faz sentido dentro de sua interpretação. Ampère preferia descrever a interação de maneira análoga à de Newton, com a força ao longo da linha reta que une os dois corpos. Ao contrário de Ørsted e outros que preferiam a explicação de um fluxo de matéria ao redor do fio, assim como Descartes usava um turbilhão no sentido do movimento dos planetas para explicar o movimento ao redor do Sol (ASSIS; CHAIB, 2011).

Já Biot e Savart consideravam a ideia de correntes moleculares desnecessária, porque não havia análogo eletrostático, magnetostático ou gravitacional para os elementos de corrente orientados. Apesar de que, tempos depois, Biot e Savart passaram a usar a ideia de um elemento de corrente proposta por Ampère.

Ampère aponta várias falhas cometidas por Biot e Savart ao buscarem uma explicação para a força de um elemento de corrente sobre um polo magnético. Ampère mostrou que "a suposição fundamental de Biot e Savart para explicar a experiência de Ørsted, baseada apenas na interação entre polos magnéticos, não deveria levar à unificação dos fenômenos magnéticos, eletromagnéticos e eletrodinâmicos" (ASSIS; CHAIB, 2011, p. 240).

\footnotetext{
${ }^{6}$ No original, “[...] that are many positive aspects related to action at a distance: its simplicity, the powerful results which are obtained with it in electromagnetism and gravitation, the implementation of Mach's principle, the fact that the first wave equation describing the propagation of electromagnetic disturbances was obtained with action at a distance laws prior to Maxwell etc" (ASSIS, 1999, p. 55).

7 No original, "[...] is undoubtedly the best, since it is the only one which makes the forces on the two elements not only equal and opposite but in the straight line which joins them" (MAXWELL, 1954, p. 174).
} 
E Grassmann, além de também criticar a complicação matemática da força de Ampère, diz que não há motivo para que a força entre dois elementos de corrente seja sempre ao longo da reta que os une.

Apesar de todas essas objeções apresentadas acima, pode-se afirmar que a força entre elementos de corrente de Ampère é uma maneira mais simples de se conceber a interação eletrodinâmica e que está de acordo com todos os resultados experimentais conhecidos. É possível descrever todos os resultados experimentais partindo do princípio de que a força está ao longo da reta que une dois elementos de corrente.

Acreditamos que com esse trabalho, seja possível observar que a eletrodinâmica de Ampère é muito consistente em todos os seus aspectos. Uma ideia tão importante e genial como essa deve ser levada em consideração quando se fala da interação eletromagnética. Ampère foi um dos maiores cientistas de sua época. Suas pesquisas sobre eletrodinâmica foram, com certeza, um dos maiores acontecimentos da história da ciência e não devem ser ignoradas.

\section{Referências}

ASSIS, A. K. T. Curso de Eletrodinâmica de Weber. Campinas: Setor de Publicações do Instituto de Física da Universidade Estadual de Campinas, 1992. Notas de Física IFGW Número 5 .

ASSIS, A. K. T. Eletrodinâmica de Weber: Teoria, aplicações e exercícios. Campinas: Editora da Unicamp, 1995.

ASSIS, A. K. T. Arguments in favour of action at a distance. In: Instantaneous Action at a Distance - "Pro" and "Contra". CHUBYKALO. A. E. et al. (Orgs.). Commack, NY: Nova Science Publishers. 1999. p. 45-56.

ASSIS, A. K. T.; CHAIB, J. P. M. de C. Nota sobre o magnetismo da pilha de Volta. Tradução comentada do primeiro artigo de Biot e Savart sobre eletromagnetismo. Cadernos de História e Filosofia da Ciência, série 3, v. 16, n. 2, p. 303-309, jul.-dez, 2006.

ASSIS, A. K. T.; CHAIB, J. P. M. de C. Eletrodinâmica de Ampère: Análise do significado e da evolução da força de Ampère, juntamente com a tradução comentada de sua principal obra sobre eletrodinâmica. Campinas, SP: Editora da Unicamp, 2011.

BUENO, M.; ASSIS, A. K. T. Cálculo de indutância e de força em circuitos elétricos. 2. ed. Montreal, Canadá: Apeiron, 2015. 
CARvalHo, B. C de. Análise Histórica do Conceito de Calor nos Trabalhos de Joule e a Transposição Didática nos Livros Didáticos de Física. 2016. 176f. Dissertação (Mestrado em Educação para a Ciência) - Universidade Estadual de Maringá, Maringá.

CHAIB, J. P. M. de C. Análise do significado e da evolução da força de Ampère, juntamente com a tradução comentada de sua principal obra sobre Eletrodinâmica. 2009. 386f. Tese (Doutorado) - Universidade Estadual de Campinas, Instituto de Física 'Gleb Wataghin'.

CHAIB, J. P. M. de C.; ASSIS, A. K. T. Distorção da obra eletromagnética de Ampère nos livros didáticos. Revista Brasileira de Ensino de Física, v. 29, n. 1, p. 65-70, 2007.

GARDELLI, D. Concepções de Interação Física: subsídios para uma abordagem histórica do assunto no ensino médio. 2004. 127f. Dissertação (Mestrado em Ensino de Ciências) - Universidade de São Paulo, São Paulo.

GARDELLI, D. Experimento de Ørsted: subsídios para uma abordagem histórica do assunto no ensino médio. 2014. 208f. Tese (Doutorado) - Universidade Estadual de Maringá, Centro de Ciências Exatas, Programa de Pós-Graduação em Educação para a Ciência e a Matemática.

GRASSMANN, H. G. A new theory of electrodynamics. In: TRICKER, R. A. R. Early Electrodynamics. London: Pergamon Press, 1965. p. 201-214.

GRIFFITHS, D. J. Eletrodinâmica. 3. ed. São Paulo: Pearson, 2010.

MARTINS, R. de A. Ørsted e a descoberta do eletromagnetismo. Cadernos de História e Filosofia da Ciência, v. 10, p. 89-114, 1986.

MARTINS, R. de A. Ørsted, Ritter and magnetochemistry. In: BRAIN, R. M.; COHEN, R. S.; KNUDSEN, O. (Eds.). Hans Christian Ørsted and the Romantic Legacy in Science: Ideas, Disciplines, Practices. New York: Springer, 2007. p. 339-385.

MAXWELL, J. C. A Treatise on Electricity \& Magnetism (1873). New York: Dover, 1954a. v. 1 .

MAXWELL, J. C. A Treatise on Electricity \& Magnetism (1873). New York: Dover, 1954b. V. 2.

SANTOS, H. S. T. dos. Análise da Lei de Biot-Savart em comparação com a força entre elementos de Ampère. 2016. 40f. Monografia (Licenciatura em Física) - Departamento de Física, Universidade Estadual de Maringá, Maringá.

ØRSTED, H. C. Experiências sobre o efeito do conflito elétrico sobre a agulha magnética. Cadernos de História e Filosofia da Ciência, v. 10, p. 115-122, 1986.

WHITTAKER, E. T. A history of the theories of aether and electricity: The Classical Theories. New York: Tomash Publishers, American Institute of Physics, 1951. 